\title{
10. Spotlight: Gene Drives - neuer Impuls für die Gentechnikkontroverse?
}

\subsection{Ausgangspunkt: Wirkprinzip und Stand der Entwicklung von Gene Drives}

Gene Drives (wörtlich übersetzt: Genantriebe) beschreiben eine vergleichsweise neue Technologie, mit der durch Erhöhung der Vererbungswahrscheinlichkeit zumindest in der Theorie ganze Populationen von sich sexuell fortpflanzenden Tieren und Pflanzen in wenigen Generationenfolgen verändert werden können. Die Idee eines Gene Drives lässt sich bis in die 1940er Jahre zu Alexander Sergeevich Serebrovskys Ansatz einer modifizierten Vererbung zur Kontrolle von Insekten wie Stubenfliegen oder Kornkäfern zurückverfolgen. Zwar können Gene Drives auch mit anderen Methoden der Genomeditierung erzeugt werden, doch nahm die Forschung zu Gene Drives erst mit der Entdeckung des CRISPR/Cas-Verfahrens im Jahr 2012 Fahrt auf, da dieses flexiblere Veränderungen des Erbguts ermöglicht als bisherige Ansätze (siehe Fehse, Kap. 6, und Fehse et al., Kap. 9).

Gene Drives zielen darauf, die Vererbungsrate eines genetischen Elementes (bspw. einer bestimmten Genvariante) gegenüber der zu erwartenden mendelschen Vererbung (von $50 \%)^{1}$ auf bis zu annähernd $100 \%$ zu erhöhen. Teilweise wird der Begriff "Gene Drive“ auch für das dieser Veränderung zu Grunde liegende „Objekt", also das genetische überdurchschnittlich häufig vererbte Element, oder als Bezeichnung für die angestrebte „Intention“, nämlich die genetische Veränderung einer Population, ver-

1 Die von Gregor Mendel 1866 beschriebenen Vererbungsregeln beziehen sich auf Merkmale, die ausschließlich von einem Gen bestimmt werden. Bei sexueller Fortpflanzung erhält ein neu entstehender Organismus von beiden Elternteilen jeweils eine Kopie (ein Allel) des entsprechenden Gens. Da die Eltern jeweils auch über zwei Allele dieses Gens verfügen, entspricht die Wahrscheinlichkeit, dass die Nachkommen eines dieser beiden Allele erben, $50 \%$. 
wendet (Alphey et al., 2020). Voraussetzungen für die Entwicklung eines Gene Drives sind sexuelle Fortpflanzung sowie schnelle Generationenfolgen (NASEM, 2016: 50).

Gene Drives bieten potenziell die Möglichkeit, durch Freisetzung weniger, mit einem Gene Drive ausgestatteter Individuen ganze Populationen zu verändern. Damit sind Gene Drives in den Augen ihrer Entwickler/-innen und Befürworter/-innen eine „Technologie, die dazu beitragen kann, einige der hartnäckigsten Probleme der Welt im Bereich von öffentlicher Gesundheit, Naturschutz und Ernährungssicherheit zu lösen“ (Alphey et al., 2020: o. S.; in ähnlicher Form: Warmbrod et al., 2020: 9). ${ }^{2}$ Von kritischen Stimmen werden Gene Drives hingegen als eine „ernste und neuartige Bedrohung ohnegleichen für die Biodiversität und die Umwelt“ (Riss et al., 2020: 1) beschrieben. ${ }^{3}$

Vor dem Hintergrund dieser gegensätzlichen Einschätzungen, welche Potenziale und Risiken mit Gene Drives einhergehen, möchten wir die wissenschaftliche und zivilgesellschaftliche Debatte zu Gene Drives vergleichen mit bisherigen Diskussionen zu Gentechnologien (zur Kontroverse um die Grüne Gentechnik siehe van den Daele, Kap. 21; Dederer, Kap. 22; Renn, Kap. 23 sowie Hampel et al., Kap. 24). Dabei gehen wir von der Ausgangsthese aus, dass sich in der Diskussion um Gene Drives zwar viele (alt) bekannte Muster finden, aber bestimmte Spezifika von Gene Drives sowohl für die Risiko- als auch für die damit verbundene Regulierungsdebatte in gewissem Maße ein Novum darstellen.

Inwieweit Gene Drives die in sie gesetzten Hoffnungen und Befürchtungen tatsächlich erfüllen (können), ist weitgehend offen. So beschränken sich praktisch erprobte Gene-Drive-Anwendungen bislang ganz überwiegend auf Insekten und Versuche fanden bisher ausschließlich im Labor statt. ${ }^{4}$ Bei einer Reihe von Experimenten zeigte sich eine (unterschiedlich starke) Resistenzbildung gegen Gene Drives, wodurch die beabsichtigte bevorzugte Vererbung des jeweiligen Elements eingeschränkt wurde (Callaway, 2017). Die meisten bislang in der Entwicklung befindlichen Gene Drives werden als „Suppression Gene Drives“ bezeichnet, durch die eine Population (bspw. mittels Erzeugung ausschließlich männlicher Nachkommen) vernichtet bzw. minimiert werden soll (NASEM, 2016: 16). Neben den supprimierenden Gene Drives existiert die zweite große

2 Eigene Übersetzung von Alphey et al.; im Original: „a technology that may help solve some of the world's most intractable problems in public health, conservation, and food security“.

3 Eigene Übersetzung von Riss et al.; im Original: „The environmental release of Gene Drive Organisms poses serious and novel threats to biodiversity and the environment at an unprecedented scale and depth".

4 Unsere Ausführungen fußen zum Teil auf drei bislang unveröffentlichten Gutachten des MaxPlanck-Instituts für Evolutionsbiologie (Reeves et al., 2020) sowie des Büros für Umweltethik (Eser, 2020), die im Auftrag des Deutschen Bundestages für das Projekt zu „Gene Drives - Technologien zur Verbreitung genetischer Veränderungen in Populationen“ des TAB erstellt wurden. 
Gruppe der „Modification Gene Drives“, die nicht auf eine Vernichtung, sondern auf eine Veränderung bestimmter Populationen zielen. ${ }^{5}$ Allerdings gibt es bisher nur wenige im Labor getestete Modification Gene Drives, da sie hinsichtlich ihrer technischen Entwicklung weit weniger ausgereift sind als bestehende Suppression Gene Drives (CSS et al., 2019: $74 \mathrm{ff}$.$) .$

\subsection{Bekannte Debattenmuster: Übersteigerte Erwartungen und Befürchtungen}

Obwohl das Anwendungsspektrum und erst recht der zukünftige mögliche Nutzen von Gene Drives weitgehend spekulativ sind und sich die Technologie noch in einer frühen Phase der Entwicklung befindet, hat sich bereits eine rege Debatte entfaltet, die stark polarisiert verläuft und altbekannte Konfliktlinien im Themenfeld der Gentechnik fortführt.

In der Diskussion um den anvisierten Einsatz von Gene Drives gegen Mückenarten, die Malaria übertragen können, zeigt sich die Polarisierung besonders deutlich. So befürchtet etwa Kevin Esvelt, einer der ersten und bekanntesten Entwickler von Gene Drives, dass mögliche externe Verzögerungen bei der Entwicklung von Gene Drives im Kampf gegen Malaria zu „Millionen von vermeidbaren Toten“ führen könnten (Esvelt, 2018: 5). Ein umgekehrtes Bild zeichnen Kritiker/-innen, die darauf hinweisen, dass Bewohner/-innen von Malariagebieten durch den beständigen Kontakt mit dem Erreger eine gewisse Immunität entwickeln. Würden Mückenpopulationen durch Gene Drives temporär reduziert, träfe der Malariaerreger nach der Entwicklung von Resistenzen auf Seiten der Vektoren auf eine weitgehend ungeschützte Bevölkerung. Dies wäre potenziell ein „katastrophaler Rückschlag“, der viele Menschen das Leben kosten würde (CSS et al., 2019: 102).

An diesem Beispiel zeigt sich zweierlei: Zum einen nennen sowohl die Befürworter/innen als auch die Gegner/-innen stichhaltige Argumente, aber es findet kein direkter Dialog untereinander statt - und es scheint, als sei dieser auch nicht gewollt. So lud bspw. die Heinrich-Böll-Stiftung zu einer öffentlichen Fachveranstaltung zu „Gene Drives - Protecting People and Nature through Genetic Extermination?“ im Herbst

5 Solche Veränderungen können beispielsweise darauf zielen, Tierarten wie Mücken genetisch so zu verändern, dass sie bestimmte Krankheiten (wie Malaria) nicht länger übertragen können. 
2020 ausschließlich kritische Expert/-innen ein. ${ }^{6}$ Dies ist selbstverständlich legitim, offenbart jedoch, dass diese noch junge Technologie bereits auf stark verhärtete Fronten trifft, die mehr übereinander als miteinander reden. Dies belegt auch der Bericht der Johns-Hopkins-Universität zu Gene Drives, bei dessen Erarbeitung 24 Experteninterviews mit Forschenden und Stakeholdern geführt wurden. Von insgesamt 13 angefragten Gene-Drive-Gegner/-innen fand keine/r sich zum Interview bereit, sodass eine kritische Perspektive weitgehend fehlte (Warmbrod et al., 2020: 16).

Das Beispiel macht zum anderen deutlich, wie stark sich beide Seiten im Bereich der Vermutungen und Hypothesen bewegen. So sind alle bislang entwickelten GeneDrive-Verfahren ausschließlich im Labor getestet worden, und ihre Auswirkungen in Wildpopulationen (etwa zum zu erwartenden Überlebens- und Fortpflanzungserfolg sowie zur Bildung von Resistenzen gegen den Gene Drive) lassen sich bislang lediglich modellieren. Ob sich tatsächlich „Millionen von Toten“ vermeiden lassen, ist daher bislang völlig offen. Vielmehr zeigen bisherige Erfolge in der lokalen Ausrottung von Malaria, dass praktisch nie ein einzelnes Mittel oder Verfahren ursächlich war, sondern eigentlich immer ein Zusammenwirken von medizinischen, sozialen und politischen Anstrengungen zum Erfolg geführt hat (Frischknecht, 2019: 27 f.). Umgekehrt basieren viele der zum Teil sehr weitreichenden Worst-Case-Szenarien von Kritiker/-innen, etwa zur Unkontrollierbarkeit der Ausbreitung von Gene Drives auch über Artgrenzen hinweg (siehe z. B. IFOAM Organics International et al., 2018), nicht auf gesichertem Wissen, sondern weitgehend auf Vermutungen oder Spekulation. Sowohl Gegner/-innen als auch Befürworter/-innen von Gene Drives neigen dazu, die zu erwartenden Funktions- und Wirkungsweisen von Gene Drives zu überhöhen, um Aufmerksamkeit für die eigenen politischen Forderungen oder die (Förderung der eigenen) Forschung zu generieren.

\subsection{Der darunter liegende Konflikt: Technologiefixierung versus Problemlösungsorientierung}

Die Überhöhung der potenziellen Wirkmächtigkeit erinnert stark an die moralisch (und sprachbildlich) aufgeladene, wohltätige Anwendung der traditionellen Gentechnik, den gentechnisch optimierten „Goldenen Reis“ zur Bekämpfung von Vitamin-Aund Eisenmangel in Entwicklungsländern (Sauter/Hüsing, 2005: 87 ff.) und verweist

6 Die Veranstaltungsankündigung der Heinrich-Böll-Stiftung findet sich hier: https://calendar. boell.de/el/node/141829 [29.01.2021], eine Zusammenfassung der Beiträge hier: https://www.stopgenedrives.eu/en/online-discussion-gene-drives-protecting-people-and-nature-through-geneticextermination/ [29.01.2021]. 
auf eine zweite Kontinuität, die die Diskussion um Gene Drives mit vorhergehenden Debatten verbindet: Der Fokus liegt auf der Technologie, ihrer Anwendung und ihren möglichen Folgen, aber nicht bzw. kaum auf den durch sie möglicherweise zu bewältigenden Herausforderungen und Problemen (z.B. durch invasive Arten auf Inseln oder durch Malaria in Subsahara-Afrika). Dadurch kommen alternative Herangehensweisen zur Problemlösung in der öffentlichen Diskussion meist viel zu kurz und es bleibt unklar, welches Chancen-Risiken-Verhältnis die neue Technologie im Vergleich zu alternativen Ansätzen bietet (Sauter, 2006). Dies mag auch damit zu tun haben, dass sowohl auf Seiten der Gegner/-innen als auch auf Seiten der Entwickler/-innen die jeweilige Expertise v. a. im Bereich der Gentechnologie liegt und nicht so sehr hinsichtlich der (Komplexität der) zu lösenden Probleme und bislang verfolgter oder alternativ in Entwicklung befindlicher Strategien. ${ }^{7}$

Beispielhaft dafür ist die Diskussion über invasive Nagetiere wie Ratten und Mäuse insbesondere auf Inseln, die als eine der zentralen Ursachen für das Artensterben unter anderem von Vögeln, Wirbellosen und Pflanzen gelten (Leitschuh et al., 2018: 122). Ein internationales Konsortium namens GBIRd (Genetic Biocontrol of Invasive Rodents) bemüht sich um die gentechnische Bekämpfung invasiver Nagetiere unter anderem mittels Gene Drives. Die weitaus größeren Schäden auf Inseln werden durch invasive Ratten im Vergleich zu Mäusen verursacht (Courchamp et al., 2003: 354 f.), aber erste Versuche zeigen, dass sich die Gene-Drive-Entwicklung für Ratten deutlich komplexer gestaltet. Folglich richten sich erste Forschungsvorhaben auf die Hausmaus, Mus musculus:

Das GBIRd-Konsortium sieht Mus musculus als den logischen Ausgangspunkt an, um ein genbasiertes Instrument zur Vernichtung invasiver Wirbeltiere zu entwickeln, zu testen sowie seine Wirksamkeit nachzuweisen. Mäuse sind eine Modellwirbeltierart für die Genforschung, sie haben eine schnelle Generationenfolge, sind klein, einfach zu halten und sind überall auf der Welt invasiv, so auch auf zahlreichen Inseln (Campbell et al., 2019: 7). ${ }^{8}$

7 Eine differenzierte Einordnung von (gen)technologischen Einzeloptionen in die aus einer Problemanalyse abgeleitete Strategienvielfalt zur Behebung von Mikronährstoffdefiziten findet sich bei Dusseldorp/Sauter (2011: 106 ff.).

8 Eigene Übersetzung von Campbell et al., im Original: „GBIRd partnership considers Mus musculus the logical starting point for developing, exploring, and providing proof-of-concept for a geneticsbased invasive vertebrate eradication tool. They are the model vertebrate species for genetics, possess a short generation-time, are small, husbandry is straight-forward, and they are invasive around the world including on many islands". 
Das Zitat verdeutlicht, dass die Auswahl des „Zielorganismus“ nicht problem-, sondern technologieorientiert erfolgt: Mäuse sind zwar weniger schädlich als andere invasive Tierarten, aber für sie scheint die Entwicklung eines Suppression Gene Drives realistischer und deshalb werden sie priorisiert. Dass Suppression Gene Drives (sofern entwickelt) das Problem invasiver Nagetiere tatsächlich wirksam und im Sinne des Artenschutzes lösen könnten, wird zugleich von anderer Seite in Frage gestellt: So errechnen Backus/Gross (2016: 7) einen Zeitraum von fünf Jahren von der Aussetzung erster Gene-Drive-Mäuse bis zur kompletten Ausrottung der Population - eventuell zu lange bei einem akuten Mäusebefall, zumal mit Giftködern ein erprobtes, wenn auch nicht unproblematisches Mittel zur Verfügung steht. Zudem müsste die Population zu Beginn um etwa ein Zehntel anwachsen (durch die zusätzlich ausgesetzten GeneDrive-Mäuse), was ebenfalls eher von Nachteil für das zu lösende Problem sein dürfte. Insofern gilt auch für die Gene-Drive-Diskussion analog zu vorherigen Debatten um gentechnische Innovationen:

Eine problemorientierte Perspektive einzunehmen, also (ergebnisoffen) zu fragen, welche (landwirtschaftlichen, umweltrelevanten, gesellschaftlichen) Probleme es eigentlich gibt und welche Technik oder andere Herangehensweise diese am besten lösen könnte - ein solches Vorgehen ist, verglichen mit technikzentrierten Debatten, aufwendig und eher unattraktiv (Sauter, 2006).

\subsection{Neue Aspekte in der Diskussion um Gene Drives: Größere Brisanz von Risikomanagement und -governance}

Mit der Polarisierung der Debatte in der Logik eines Freund-Feind-Schemas sowie der Technologiezentrierung weist die Gene-Drive-Debatte zwei Gemeinsamkeiten mit bisherigen gentechnologischen Diskursen auf. Gleichwohl zeigen sich, ausgehend von bestimmten Spezifika von Gene Drives, sowohl in der Risiko- als auch in der damit verbundenen Regulierungsdebatte in gewissem Maße auch neue Aspekte. Gene Drives zielen auf eine Anwendung in Wildpopulationen, außerhalb von menschlich kontrollierten oder kultivierten Ökosystemen. Damit bilden sie einen fundamentalen, inhärenten Gegensatz zu bisherigen Gentechnikanwendungen in der Landwirtschaft, bei denen seit jeher die Verhinderung einer Ausbreitung in umliegende Naturräume die zentrale und kaum umstrittene Aufgabe des Risikomanagements war (Sauter/Meyer, 2000). Noch stärker - und nicht begrenzt auf Personen oder Institutionen mit einer gentechnikkritischen oder zumindest -skeptischen Haltung - als bei bisherigen Debatten 
um Gentechnik richten sich deshalb zentrale Bedenken auf Umwelt- und Naturschutzrisiken, insbesondere auf die potenziell irreparable Schädigung von Ökosystemen. An diese Bedenken knüpfen auch aktuelle repräsentative Befragungen der europäischen Bevölkerung an, in denen in acht verschiedenen Staaten zwischen zwei Drittel und vier Fünftel aller Befragten der Aussage zustimmten, dass Gene-Drive-Organismen so lange nicht freigesetzt werden sollten, bis ihre Unschädlichkeit unter anderem für die Biodiversität nachgewiesen sei (DNR et al., 2021).

Auch die Entwickler von Gene Drives haben diese Bedenken als zentral erkannt und setzen deshalb beträchtliche Energien in die Entwicklung von „genetischen Rückholsystemen“ („Reversal Drives“) bzw. von sich selbst abschwächenden Gene Drives („Threshold“ und „Daisy-Chain-Drives“; Warmbrod et al., 2020). Die Nutzung solcher biologischen Einhegungs- bzw. Containmentmaßnahmen (in Form der Beeinflussung der Reproduktionseigenschaften) war bislang v. a. bei gentechnisch veränderten Pflanzen zur Produktion von Arzneimittelinhaltsstoffen, sog. „Plant Made Pharmaceuticals“ (PMP), die anders als Lebens- oder Futtermittelpflanzen prinzipiell als mit einem gewissen Risiko behaftet angesehen werden, in Erwägung gezogen bzw. verfolgt worden (Sauter/Hüsing, 2005: 196 ff.). Dabei war und ist zu bedenken, dass biologische Systeme aufgrund ihrer Offenheit, Variabilität und Veränderungsfähigkeit de facto nie eine hundertprozentige „Sicherheit“ bieten können. Daher wurde auch hinsichtlich eines vollständigen Containments von PMP gefolgert, dass ein solches nur in Kombination mit physikalischen Maßnahmen, also letztlich in einem geschlossenen System, gewährleistet werden kann (Sauter/Hüsing, 2005: 220, unter Verweis auf Pohl/Arndt, 2004: 72). Die mangelnde Kontrollierbarkeit ist auch eine fundamentale Sorge bei der Freisetzung gentechnisch veränderter Tiere und hat Freilandversuche in Europa bislang verhindert.

Wenn ein biologisches oder physikalisches Containment, wie bei Gene Drives, aus prinzipiellen, immanenten Gründen keine Option darstellt, weil ihr potenzieller Erfolg gerade auf der Unkontrollierbarkeit der Ausbreitung beruht, dann muss das damit verbundene Risiko, bspw. der Übertragung auf verwandte Arten, zum einen durch den erhofften hochrangigen Nutzen (etwa mit Blick auf die Vermeidung von über 400.000 Malariatoten pro Jahr, mehrheitlich Kinder unter 5 Jahren) klar aufgewogen werden und zum anderen erhalten grenzübergreifende Maßnahmen und Abkommen zum Umgang mit der Ausbreitung von Gene-Drive-Organismen eine deutlich größere Relevanz und Brisanz als bislang beim Handel mit bzw. der Einfuhr von „konventionellen“ gentechnisch veränderten landwirtschaftlichen Nutzpflanzen. Bisherige internationale Abkommen wie das Cartagena-Protokoll über die biologische Sicherheit und das Übereinkommen über die biologische Vielfalt („Convention on Biological Diversity“, CBD) 
schließen zwar eine Einfuhr von lebenden veränderten Organismen ohne die vorherige explizite Einwilligung des betroffenen Staates aus, aber die USA als einer der Vorreiter in der Gene-Drive-Entwicklung sind gar nicht Unterzeichner dieser Abkommen. Dies stellt eine große Lücke in der internationalen Governance dar (Warmbrod et al., 2020: 51; NASEM, 2016: 172). Der Notwendigkeit internationaler Abstimmung kommt im Fall von Gene Drives im Vergleich zu vorherigen gentechnischen Neuerungen folglich besonderes Gewicht zu.

\subsection{Ausblick: Herausforderungen für demokratische Mitsprache in neuer Dimension}

In der Zusammenschau wird deutlich, dass die Diskussion um Gene Drives zwar mit Blick auf ihre Polarisierungstendenz und ihren Technologiefokus einerseits in der Kontinuität bisheriger Gentechnikdiskurse steht, zugleich jedoch das Grundprinzip der autonomen Verbreitung in Wildpopulationen ein absolutes Novum bei gentechnischen Anwendungen darstellt. Die daraus resultierenden Unwägbarkeiten hinsichtlich des Containments der Technologie führt die Wichtigkeit grenzübergreifender Maßnahmen und Abkommen zum Umgang mit einer möglichen Ausbreitung von Gene-Drive-Organismen bereits im derzeitigen Erforschungs- und bevorstehenden Erprobungsstadium vor Augen. Dabei stellen sich mindestens zwei große Herausforderungen: Zum einen sind die USA, als einer der führenden Forschungs- und Entwicklungsstandorte, kein Unterzeichnerstaat des Cartagena-Abkommens; und zum anderen besteht ein technologie- und entwicklungspolitisches Spannungsfeld zwischen den Ländern, in denen der überwiegende Teil der Forschung stattfindet (Industrieländer wie die USA, Großbritannien, Australien und Neuseeland), und denjenigen, in denen voraussichtlich die am weitesten vorangeschrittenen Gene-Drive-Systeme erprobt werden sollen, nämlich die afrikanischen Staaten Burkina Faso, Ghana, Mali und Uganda. ${ }^{9}$ Es stellen sich grundlegende und konkrete Fragen nach demokratischer Mitsprache und Kontrolle durch die potenziell betroffene Bevölkerung. Dass mit Target Malaria das größte Forschungskonsortium zum Einsatz von Gene-Drive-Mücken in der Malariabekämpfung diese Herausforderung mindestens mit Blick auf die öffentliche Wahrnehmung erkannt hat und zu adressieren versucht (Barry et al., 2020), kann als positives Zeichen dafür interpretiert werden, dass zumindest in dieser Hinsicht das Problembewusstsein - gegenüber einer eindimensionalen Technologiefixierung - gewachsen ist.

9 Diese Länder nennt Target Malaria als ihre Einsatzgebiete auf ihrer Webseite unter dem Reiter „Where We Operate“, siehe unter: https://targetmalaria.org/ [11.02.2021]. 


\subsection{Literaturverzeichnis}

Alphey, L. et al. (2020): Opinion: Standardizing the definition of gene drive. In: Proceedings of the National Academy of Sciences of the United States of America 117(49): 30864-30867.

Backus, G./Gross, K. (2016): Genetic engineering to eradicate invasive mice on islands: modeling the efficiency and ecological impacts. In: Ecosphere 7(12), Online-Publikation 27.12.2016. DOI: 10.1002/ ecs2.1589.

Barry, N. et al. (2020): Motivations and expectations driving community participation in entomological research projects: Target Malaria as a case study in Bana, Western Burkina Faso. In: Malaria Journal 19(1): 199-209.

Callaway, E. (2017): Gene drives thwarted by emergence of resistant organisms. In: Nature 542(7639): 15.

Campbell, K. et al. (2019): A potential new tool for the toolbox: assessing gene drives for eradicating invasive rodent populations. In: Veitch, C. et al. (Hrsg.): Island invasives: scaling up to meet the challenge. Proceedings of the international conference on island invasives 2017. Occasional Paper SSC no. 62, Gland: 6-14.

Courchamp, F. et al. (2003): Mammal invaders on islands: impact, control and control impact. In: Biological reviews of the Cambridge Philosophical Society 78(3): 347-383.

CSS = Critical Scientists Switzerland et al. (2019): Gene drives. A report on their science, applications, social aspects, ethics and regulations. Bern.

DNR = Deutscher Naturschutzring et al. (2021): Umfrage: Mehrheit von EU Bürger*innen lehnt gentechnische Veränderung von wildlebenden Arten ab. Unter: https://www.dnr.de/presse/ pressemitteilungen/pm-2020/umfrage-mehrheit-von-eu-buergerinnen-lehnt-gentechnischeveraenderung-von-wildlebenden-arten-ab/ [29.01.2021].

Dusseldorp, M./Sauter, A. (2011): Forschung zur Lösung des Welternährungsproblems - Ansatzpunkte, Strategien, Umsetzung. TAB-Arbeitsbericht Nr. 142, Büro für Technikfolgen-Abschätzung beim Deutschen Bundestag, Berlin.

Esvelt, K. (2018): Gene drive technology: The thing to fear is fear itself. In: Editing Biosecurity 4, George Mason University.

Frischknecht, F. (2019): Malaria. Tödliche Parasiten, spannende Forschung und keine Impfung. Essentials, Wiesbaden.

IFOAM Organics International et al. (2018): A call to protect food systems from genetic extinction technology: The global food and agriculture movement says NO to release of gene drives. Unter: https://www.organicseurope.bio/content/uploads/2020/06/call-_gene_drives_and_agriculture_0. pdf?dd [29.01.2021].

Leitschuh, C. et al. (2018): Developing gene drive technologies to eradicate invasive rodents from islands. In: Journal of Responsible Innovation 5(1): S121-S138. 
NASEM (2016): Gene drives on the horizon: Advancing science, navigating uncertainty, and aligning research with public values. Report by the National Academies of Sciences, Engineering, and Medicine. The National Academies Press, Washington (DC).

Pohl, M./Arndt, N. (2004): Containment, Confinement und Koexistenz beim Molecular Farming Möglichkeiten und Probleme. Gutachten im Auftrag des Deutschen Bundestages, TÜV NORD EnSys Hannover GmbH \& Co. KG, Hannover (unveröffentlicht).

Riss, J. et al. (2020): Open letter to the EU commission: Please support a global moratorium on the environmental release of gene drive organisms. Unter: https://update.zs-intern.de/fileadmin/ files/sos/gene_drive/Open_Letter_to_the_EU_Commission_Please_support_a_global_ moratorium_on_the_release_of_Gene_Drive_Organisms_30.06.2020.pdf [29.01.2021].

Sauter, A. (2006): Chancen und Grenzen der grünen Gentechnologie. Vortrag im Rahmen der Konferenz zu „Gentechnik - Top oder Flop?“ der Rosa-Luxemburg-Stiftung und des Bildungsvereins Elbe-Saale, Halle/Saale.

Sauter, A./Hüsing, B. (2005): TA-Projekt Grüne Gentechnik - Transgene Pflanzen der 2. und 3. Generation. TAB-Arbeitsbericht Nr. 104, Büro für Technikfolgen-Abschätzung beim Deutschen Bundestag, Berlin.

Sauter, A./Meyer, R. (2000): Risikoabschätzung und Nachzulassungs-Monitoring transgener Pflanzen. TAB-Arbeitsbericht Nr. 68, Büro für Technikfolgen-Abschätzung beim Deutschen Bundestag, Berlin.

Warmbrod, K. et al. (2020): Gene drives: Pursuing opportunities, minimizing Risk. Johns Hopkins Center for Health Security, Baltimore. 